\title{
Protein Structure Prediction Using Hybrid AI Methods"
}

X. Guan, R. J. Mural', E. C. Uberbacher

\section{Informatics Goup \\ Intelligent Systems Section}

Engineering Physics and Mathematics Division and 'Biology Division

Oak Ridge National Laboratory

P.O. Box 2008

Oak Ridge, Tennessee 37831-6364

\section{DISCLAIMER}

\begin{abstract}
This report was prepared as an account of work sponsored by an agency of the United States Government. Neither the United States Government nor any agency thereof, nor any of their employees, makes any warranty, express or implied, or assumes any legal liability or responsibility for the accuracy, completeness, or usefulness of any information, apparatus, product, or process disclosed, or represents that its use would not infringe privately owned rights. Reference herein to any specific commercial product, process, or service by trade name, trademark, manufacturer, or otherwise does not necessarily constitute or imply its endorsement, recommendation, or favoring by the United States Government or any agency thereof. The views and opinions of authors expressed herein do not necessarily state or reflect those of the United States Government or any agency thereof.
\end{abstract}

Submitted to:

The Tenth IEEE Conference on Artificial Intelligence for Applications, Marriott Riverwalk, San Antonio, Texas, March 1-4, 1994

\footnotetext{
Research was support by the Office of Health and Environmental Research, U. S. Department of Energy under Contract No. DE-AC05-84OR21400 with Martin Marietta Energy Systems, Inc.

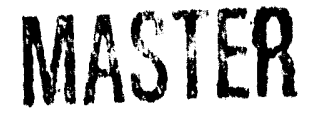

DISTRIBUTION OF THIS DOCUMENT IS UNLIMITED 


\title{
Protein Structure Prediction Using Hybrid AI Methods*
}

\author{
X. Guan, R. J. Mural' ${ }^{1}$ E. C. Uberbacher \\ Engineering Physics and Mathematics Division \\ and 'Biology Division \\ Oak Ridge National Laboratory \\ Oak Ridge, TN 37831-6364
}

\begin{abstract}
This paper describes a new approach for predicting protein structures based on Artificial Intelligence methods and genetic algorithms. We combine nearest neighbor searching algorithms, neural networks, heuristic rules and genetic algorithms to form an integrated system to predict protein structures from their primary amino acid sequences. First we describe our methods and how they are integrated, and then apply our methods to several protein sequences. The results are very close to the real structures obtained by crystallography. Parallel genetic algorithms are also implemented.

$\begin{array}{ll}\text { TOPIC: } & \text { Search, genetic algorithm } \\ \text { DOMAIN: } & \text { Computational biology } \\ \text { LANGUAGE: } & \text { C } \\ \text { STATUS: } & \text { Under development } \\ \text { EFFORT: } & \text { 2 person years } \\ \text { IMPACT: } & \text { Demonstrated suitability of Ar- } \\ & \text { tificial Intelligence based system } \\ & \text { for solving the problem of protein } \\ & \text { structure prediction from protein } \\ & \text { sequence data from the Human } \\ & \text { Genome Project }\end{array}$
\end{abstract}

\footnotetext{
- Research was supported by the Office of Health and Environmental Research, U.S. Department of Energy under Contract No. DE-AC05.84OR21 400 with Martin Marietta Energy System, Inc.
}

\section{Introduction}

The Human Genome Project will produce a huge amount of DNA and protein sequences. In order to analyze the information contained in these sequences. efficient computational tools have to be constructed to process them as they are produced. The first step is to locate the genes in newly sequenced DNA. An example is the GRAIL system [1, 2], an integrated artificial intelligence system combing multi-sensor neural networks and parallel processing tools to recognize and interpret genes in DNA sequences. The second step will be to develop methods to predict and model the structure and function of the proteins coded by the genes.

It is known that the function of a protein depends on its structure. Currently there are several thousand known protein sequences. Only about 120 unique protein structures have been determined through crystallography, which is extremely labor and time intensive. As the Human Genome Project develops, protein sequences will be produced at an increasing rate. The gap between the number of the known protein sequences and the number of the known protein structures will become wider and wider.

A protein sequence is a chain of amino acids, occurring in twenty natural forms. Protein structure can be described in three levels (see Figure 1). The primary sequence of a protein is just a linear sequence of the amino acids. The secondary structure is a higher level of description of the protein where segments of the primary sequences form regular structures. Three most commonly used classes of secondary structures are $\alpha$-helix, $\beta$-strand, and coil. The tertiary structure is the three dimensional structure of a protein sequence, or fully folded protein sequence. The principles 
of how protein sequences form secondary structure and tertiary structure from their primary sequences are not well understood.

$$
\begin{gathered}
\text { Val - Ala - Arg - Ser - Asn - Phe - Asn ...... } \\
\text { Primary }
\end{gathered}
$$

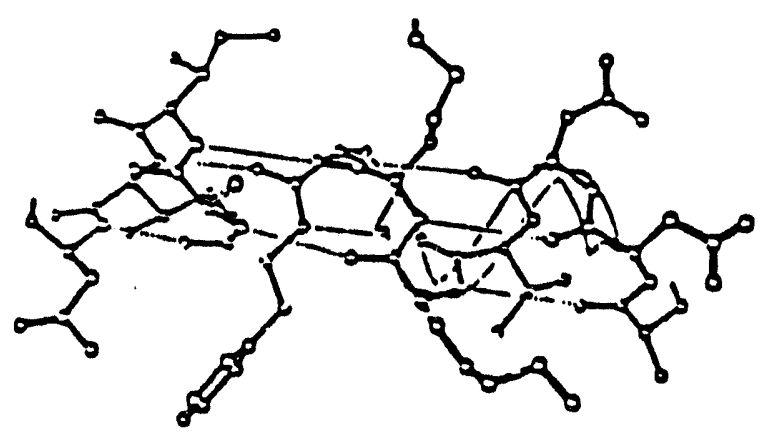

Secondary

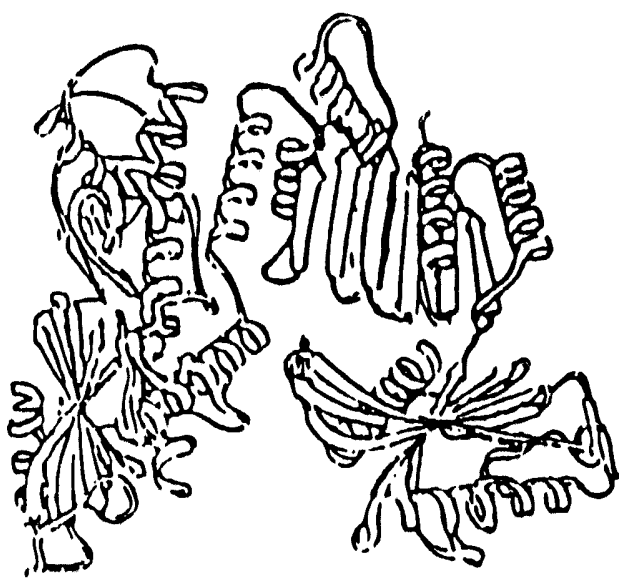

Tertiary

Figure 1. Three level description of a protein sequence.

There has been significant research into efficient computational methods to predict protein structure information. Several researchers [3-6] have used neural network and statistical methods to predict secondary structures from primary sequences. If accurate secondary structures of a protein can be obtained, they can be used to help derive the tertiary folding pattern of the sequence. Limited success has been achieved despite intensive research in this area for more than a decade. It is believed that methods for prediction of long range interactions and a larger database of known protein structures would be required before further progress can be made [7].

There are also methods which predict a protein's tertiary structure directly from its sequence. One approach relies on the homology between proteins; structure information is derived from similar sequences with known structures [8], and is limited by the degree to which proteins exhibit homology at the primary sequence level. Sometimes different protein sequences (with low percentage of homology) may have similar structures. In this case, the homology-based algorithms may not be effective.

Another approach to direct structure prediction [9] has been to use Memory Based Reasoning (MBR) to predict the torsion angles $\phi$ and $\psi$ of amino acids on a Connection Machine. With running time of 100 hours on the Connection Machine, the overall errors were $\phi_{\text {error }}=37.7^{\circ}$ and $\psi_{\text {error }}=63.7^{\circ}$.

Molecular dynamics approaches for protein structure calculation are very computationally intensive and can only be used to simulate relatively short period of time of the protein folding process.

In this paper, we describe a new approach using a combination of nearest neighbor search methods, a neural network, heuristic rules, and genetic algorithms to predict three dimensional structures of proteins. The unique aspect of our method is that it does not depend solely on the homology or definite secondary structures to predict three dimensional structure. We use homology-derived information to form probable secondary structures in the form of torsion angle probability distribution and subsequently a genetic algorithm to search for the optimal solution, guided by the torsion angle probability distribution and other biological knowledge represented as a fitness function in the genetic algorithm.

\section{Methods}

The basic three dimensional folding pattern of a protein sequence can be obtained if the two backbone torsion angles $(\phi$ and $\psi$ ) per amino acid residue are known (see Figure 2). We have therefore chosen to use a sequence of $\phi-\psi$ angles corresponding to the amino acid residues of a protein sequence to represent the protein's 
structure.

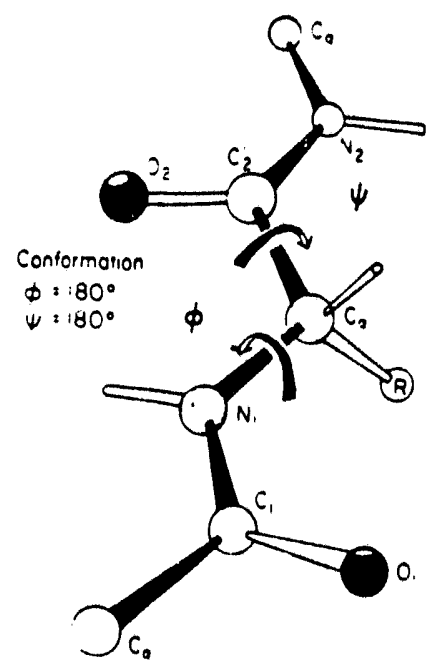

Figure 2. $\phi$ and $\psi$ angles on the backbone of a protein.

Given a known protein sequence, nearest neighbor search methods and a neural network are used to find close matches of each local sequence region in the protein database. Our approach here is to achieve greater generalization of structural principles by using the physical properties of residues as the parameters rather than the sequence itself.

There are a number of important physical properties of the amino acids that are believed to affect the three dimensional folding of proteins. We have used five based on an analysis done by Heringa and Argos [10]: hydrophobicity, turn preference, residue bulk, refractivity index and anti-parallel strand preference. A window of fixed size is used around an amino acid to predict the amino acid's torsion angles. Within the window, different properties and some combinations of them are used to measure the distance between the sequence in the window and others from sequences in the protein database. To demonstrate the performance of this search method, we have calculated the torsion angles from the top 10 matches in the database search, and drawn the predicted structure and real structure for a segment of 16 residues (see Figure 3 ). The nearest neighbor search together with the neural network produces fairly close prediction of the real structure although some $\psi$ angles are in the wrong domain.

Despite the fact that $\phi$ and $\psi$ can be predicted by this method, we do not use definite secondary structures or fixed $\psi$ and $\phi$ angles in the prediction of tertiary folding. Instead we calculate a 2-dimensional $\phi$ and $\psi$ probability distribution for each amino acid residue of the sequence. 'The distributions are used in a later stage of the computation to help direct the search of the global optimal solution using a genetic algorithm. Genetic algorithms are designed to explore a huge solution space efficiently and to combine local partial solutions to form a global optimal solution. In a given member of the population, any segments which have a correct prediction of torsion angles may serve as the building blocks in the genetic algorithm search space. A score is obtained for each model based on the torsion angle distribution, together with other constraints to guide and constrain the genetic algorithm search.

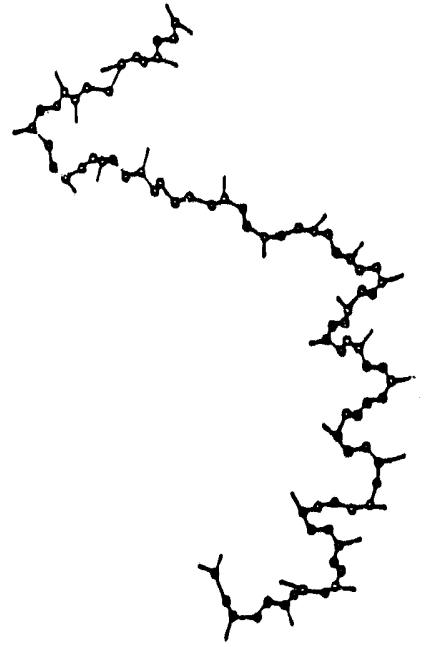

Predicted Structure

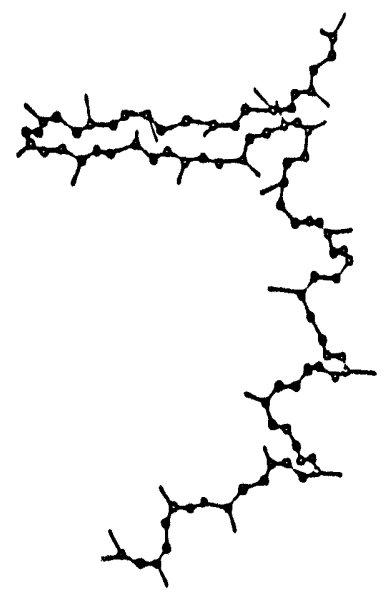

Real Structure
Figure 3. Real structure and predicted structure from the nearest neighbor search.

Figure 4 shows examples of two dimensional $\phi-\psi$ probability distributions which have been determined for 7 residue window size. In each example, the distribution is for the middle residue of the 7 residue window, where $\phi$ is along the $\mathrm{X}$ axis and $\psi$ along $\mathrm{Y}$ axis, in the range of $-180^{\circ}$ to $+180^{\circ}$ in increments of $30^{\circ}$. 


$\begin{array}{rllllllllllllll}\text { ial - Aia - Arg - Ser - Asn - Phe - Asn } & & \\ & 2 & 0 & 1 & 2 & 0 & 0 & 0 & 0 & 0 & 0 & 0 & 0 & 0 \\ & 2 & 2 & 2 & 1 & 0 & 0 & 0 & 0 & 0 & 0 & 0 & 0 & 0 \\ 0 & 0 & 1 & 0 & 0 & 0 & 0 & 0 & 0 & 0 & 0 & 0 & 0 \\ 0 & 1 & 0 & 1 & 0 & 0 & 0 & 0 & 0 & 0 & 0 & 0 & 0 \\ 0 & 0 & 0 & 0 & 1 & 0 & 0 & 0 & 0 & 0 & 0 & 0 & 0 \\ 0 & 0 & 0 & 0 & 0 & 2 & 1 & 0 & 0 & 0 & 0 & 0 & 0 \\ 0 & 0 & 1 & 1 & 20 & 1 & 0 & 0 & 0 & 0 & 0 & 0 & 0 \\ 0 & 0 & 0 & 1 & 2 & 0 & 0 & 0 & 0 & 0 & 0 & 0 & 0 \\ 0 & 0 & 2 & 0 & 0 & 0 & 0 & 0 & 1 & 1 & 0 & 0 & 0 \\ 0 & 0 & 0 & 0 & 0 & 0 & 0 & 0 & 0 & 0 & 0 & 0 & 0 \\ 1 & 0 & 0 & 0 & 0 & 0 & 0 & 0 & 1 & 0 & 0 & 0 & 1 \\ 0 & 0 & 0 & 3 & 0 & 0 & 0 & 0 & 0 & 0 & 0 & 0 & 0\end{array}$

Probably alpha

Gly - Ile - Ile - Ile - Pro - Gly - Ala

\begin{tabular}{l|llllllllllll}
0 & 1 & 1 & 5 & 1 & 0 & 0 & 0 & 0 & 0 & 0 & 0 & 0 \\
0 & 1 & 3 & 0 & 0 & 0 & 0 & 0 & 0 & 0 & 0 & 0 & 0 \\
0 & 1 & 3 & 0 & 0 & 0 & 0 & 0 & 0 & 0 & 0 & 0 & 0 \\
0 & 0 & 0 & 0 & 1 & 0 & 0 & 1 & 0 & 0 & 0 & 0 & 0 \\
0 & 0 & 1 & 0 & 0 & 0 & 0 & 0 & 0 & 0 & 0 & 0 & 0 \\
0 & 0 & 0 & 0 & 0 & 0 & 0 & 0 & 0 & 0 & 0 & 0 & 0 \\
0 & 0 & 0 & 0 & 3 & 0 & 0 & 0 & 0 & 0 & 0 & 0 & 0 \\
0 & 0 & 0 & 3 & 0 & 0 & 1 & 0 & 0 & 0 & 0 & 0 & 0 \\
0 & 0 & 0 & 1 & 1 & 0 & 0 & 0 & 0 & 1 & 0 & 0 & 0 \\
0 & 0 & 0 & 2 & 0 & 0 & 0 & 0 & 0 & 1 & 0 & 0 & 0 \\
0 & 0 & 0 & 0 & 0 & 0 & 0 & 0 & 3 & 0 & 0 & 0 & 0 \\
0 & 0 & 0 & 0 & 1 & 0 & 0 & 0 & 0 & 0 & 0 & 0 & 0 \\
1 & 1 & 4 & 1 & 0 & 0 & 0 & 0 & 0 & 0 & 0 & 0 & 1
\end{tabular}

Possibly Beta

Figure 4. Examples of $\phi-\psi$ distribution.

The protein structures were taken from Protein Database Bank (PDB). As is commonly done, we use a homology search algorithm to remove sequences that are redundant and exclude examples with homology to test structures.

\section{Genetic Algorithms}

A common problem associated with protein folding methods is that the potential solution space is huge, therefore the computation is enormous. Genetic alyorithms make it possible to explore a far greater range of potential solutions than conventional methods[10]. When dealing with a problem such as protein folding where many local minima exist, the genetic algorithm's implied parallelism allows one to explore many local minima in parallel while manipulating relatively few data.
In our system, a protein trial structure (model) is repsesented by a sequence of torsion angles corresponding to its amino acids. We start with a population of models generated from the $\phi-\psi$ distributions using a Monte Carlo method. The models are ranked according to their fitness. Top ranking models are crossed over and mutated to produce the next generation.

The foundation of the genetic algorithm is the development of a proper fitness function. Our fitness function is based on the sum of a number of empirical energy terms. Several of these are standard empirical energy terms used in macromolecular energy minimizations; terms for Van der Waals interaction, Coulombic interaction, hydrogen bonding (modeled as a modified Van der Waals term), and an empirical term for hydrophobicity which is based on solvent accessibility to simplified sidechains. The details of these terms are beyond the scope of this paper. These empirical energies are combined with the torsion "energy" term based on probabilistic information (derived from agreement with predicted $\phi-\psi$ distributions for each amino acid). Torsion energy is traditionally calculated from detailed atomic positions, but this would require detailed knowledge of sidechain geometry and conformation. Our approach eliminates the requirement for this knowledge and streamlines the calculation since we do not model the sidechain in detail.

The different terms in the fitness function must be properly balanced. Each has a weight coefficient which has been tuned by trial and error, and by applying intuition gained from structures resulting from the genetic algorithm. The more standard empirical energies (Coulombic, Hydrogen bonding and Van der Waals interactions) required only small changes from published values. Torsion and hydrophobic energies, which are obtained here by non-standard means, required sig. nificant tuning.

\section{Parallel Genetic Algorithm}

We have also implemented the above genetic algorithm on a Intel $\mathrm{iPSC} / 860$ parallel computer. Initially each node has a sub-population of protein models on its local memory. Each node proceeds like the sequential version for a few generations, then top models from each node are periodically exchanged among the nodes to cross-fertilize the whole population and to maintain diversity in the whole population. With the parallel genetic algorithms, structures of large protein sequences can potentially be calculated. 


\section{Results}

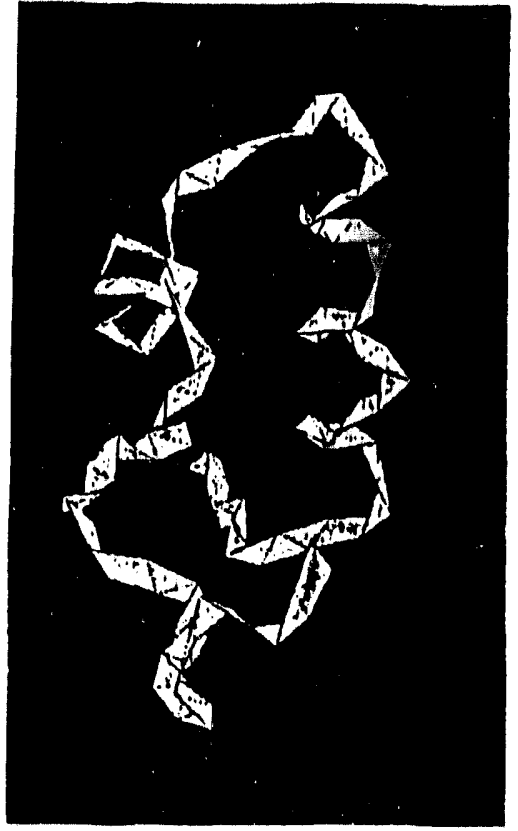

Real structure

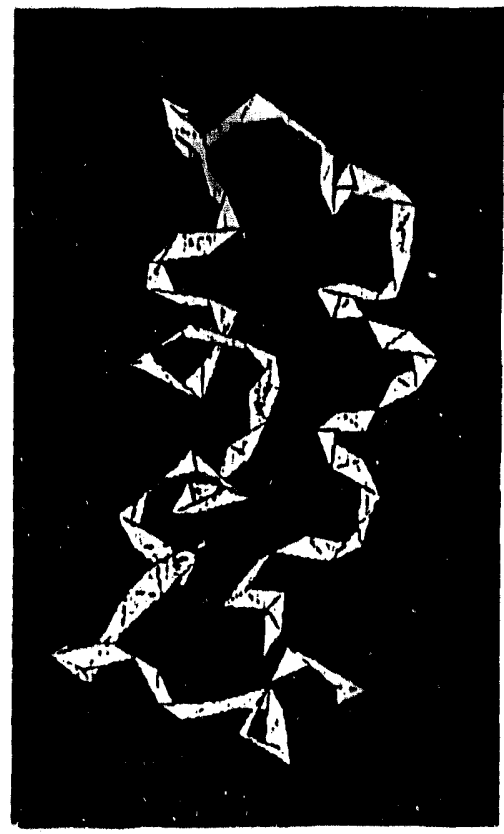

Predicted structure

Figure 5. Real and predicted structures.
We have applied our method to Crambin, a protein with 46 residues. Its folding pattern involves several secondary structures and their interactions. Displayed in ribbon style (figure 5) are the real and predicted structures.

\section{Conclusions}

An integrated Al system has been developed for protein structure prediction. Nearest neighbor searching algorithms are used to find the close matches of a local region of a protein sequence in the prot in database and the probability distribution of the torsion angles are calculated. A genetic algorithm is used to search for the optimal folding of the protein sequence based on the torsion angle distribution and other biological knowledge represented as the fitness function for the genetic algorithm. The application of the integrated system for several small protein sequences results in fairly close predictions of the structures compared to the real structures.

\section{References}

[1] E. C. Uberbacher and R. J. Mural, "Locating protein-coding regions in human DNA sequences by a multiple sensor-neural network approach," Proc. Natl. Acad. Sci. USA, vol. 88, 11261-11265.

[2] X. Guan, Richard Mural, Reinhold Mann, Ralph Einstein, and Edward Uberbacher, "GRAIL: An Integrated Artificial Intelligence System for Gene Recognition and Interpretation," Proc., The Eighth IEEE Conference on Artificial Intelligence for Applications, 1992, 9-13.

[3] P. Y. Chou and G. D. Fasman, "Prediction of the Secondary Structure of Proteins from Their Amino Acid Sequences," Advan. Enzymol., 47, 1978, 44148.

[4] N. Qian and T. J. Sejnowski, "Predicting the Secondary Structure of Globular Proteins Using Neural Network Models," Journal of Molecular Biology, $202,1988,865-884$.

[5] D. G. Kneller F, E. Cohen, and R. Langridge, "Improvements in Protein Secondary Structure Prediction by an Enhanced Neural Network," Journal of Molecular Biology, 214, 1990, 171-182. 
[6] Paul Stolorz, Alan Lapedes, and Yuan Xia, "Predicting Protein Secondary Structure Using Neural Net and Statistical Methods," Journal of Molecular Biology, 225, 1992, 363-377.

[7] M. J. Rooman and S. J. Wodak, "Weak Correlation between Predictive Power of Individual Sequence Patterns and Overall Prediction Accuracy in Proteins," PROTEIN: Structure, Function and Genet. ics, 9, 1991, 69-78.

[8] Chris Sander and Reinhard Schneider, "Database of Homology-Derived Protein Structures and the Structural Meaning of Sequence Alignment," PRO. TEIN: Structure, Function and Genetics, 9, 1991, 56-68.

[9] X. Zhang, David Waltz and J. P. Mesirov, "Protein Structure Prediction by A Data-level Parallel Algorithm," Proc., International Conference on Parallel Processing, 1989, 215-223.

[10] J. H. Holland, "Genetic Algorithm," Scientific American, July, 1992, 66-72.

[11] Jaap Heringa and Patrick Argos, "Side-Chain Clusters in Protein Structures and Their Role in Protein Folding," Journal of Molecular Biology, 220, 1991, 151-171. 


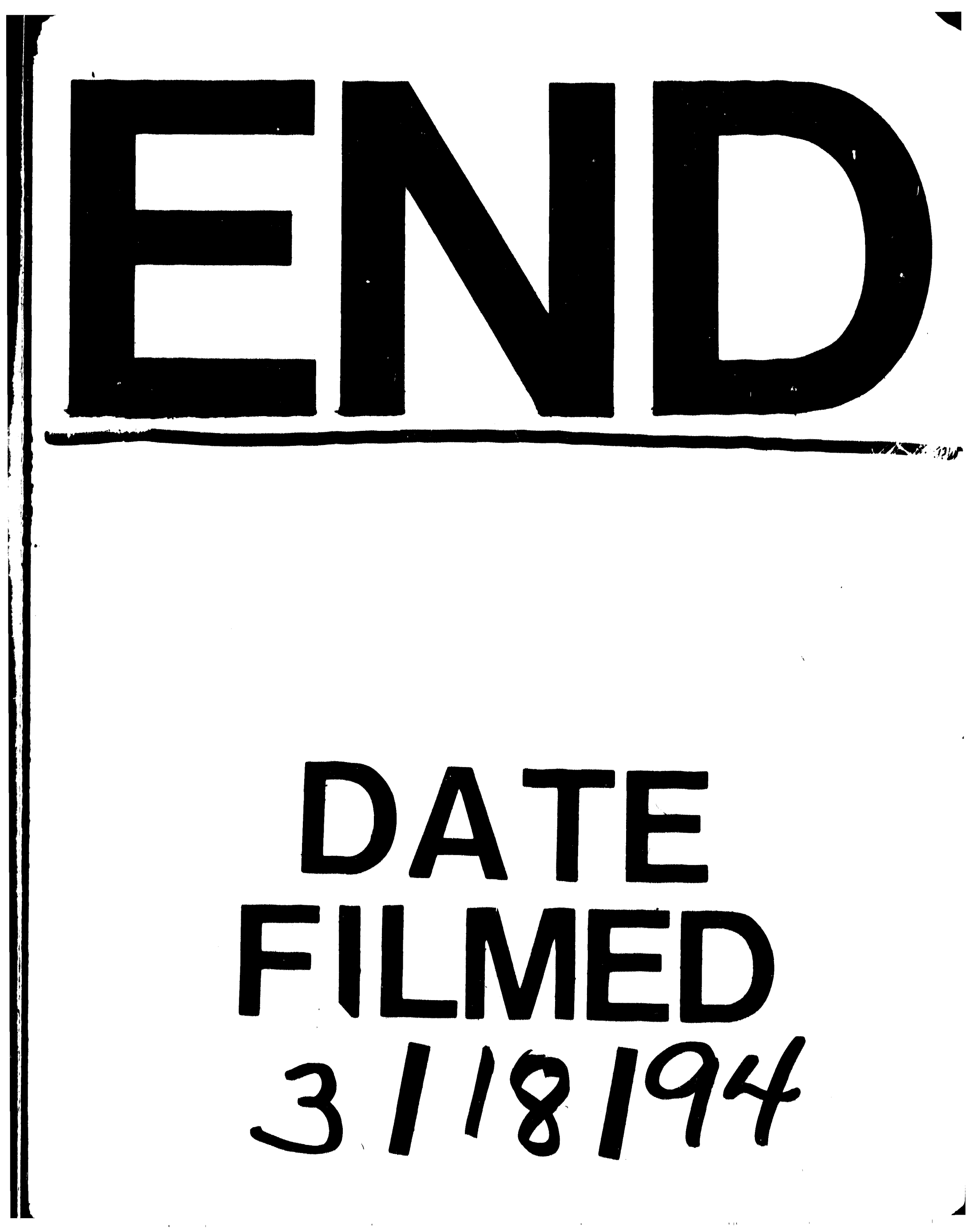


